\title{
Potential Implications of Slim Modiolar Electrodes for Severely Malformed Cochleae: A Comparison With the Straight Array With Circumferential Electrodes
}

\author{
Sang-Yeon Lee ${ }^{1}$ (i) $\cdot$ Byung Yoon Choi ${ }^{2}$ (i) \\ ${ }^{I}$ Department of Otorhinolaryngology-Head and Neck Surgery, Seoul National University Hospital, Seoul; \\ ${ }^{2}$ Department of Otorhinolaryngology-Head and Neck Surgery, Seoul National University Bundang Hospital, Seongnam, Korea
}

\begin{abstract}
Objectives. Malformations of the inner ear account for approximately $20 \%$ of cases of congenital deafness. In current practice, straight arrays with circumferential electrodes (i.e., full-banded electrodes) are widely used in severely malformed cochleae. However, the unpredictability of the location of residual spiral ganglion neurons in such malformations argues against obligatorily using full-banded electrodes in all cases. Here, we present our experience of electrically evoked compound action potential (ECAP) and radiography-based selection of an appropriate electrode for severely malformed co-
\end{abstract} chleae.

Methods. Three patients with severely malformed cochleae, showing cochlear hypoplasia type II (CH-II), incomplete partition type I (IP-I), and cochlear aplasia with a dilated vestibule (CADV), respectively, were included, and the cochlear nerve deficiency (CND) was evaluated. A full-banded electrode (CI24RE(ST)) and slim modiolar electrode (CI632) were alternately inserted to compare ECAP responses and electrode position.

Results. In patient 1 (CH-II with CND), who had initially undergone cochlear implantation (CI) using a lateral wall electrode (CI422), revision CI was performed due to incomplete insertion of CI422 and resultant unsatisfactory performance by explanting the CI422 and re-inserting the CI24RE(ST) and CI632 sequentially. Although both electrodes elicited reliable ECAP responses with correct positioning, CI24RE(ST) showed overall lower ECAP thresholds compared to CI632; thus, CI24RE(ST) was selected. In patient 2 (IP-I with CND), CI632 elicited superior ECAP responses relative to CI24RE(ST), with correct positioning of the electrode; CI632 was chosen. In patient 3 (CADV), CI632 did not elicit an ECAP response, while meaningful ECAP responses were obtained with the CI24RE(ST) array once correct positioning was achieved. All patients' auditory performance markedly improved postoperatively.

Conclusion. The ECAP and radiography-based strategy to identify an appropriate electrode may be useful for severely malformed cochleae, leading to enhanced functional outcomes. The practice of sticking to full-banded straight electrodes may not always be optimal for IP-I and CH-II.

Keywords. Cochlear Implantation; Cochlea; Congenital Abnormalities; Electrodes

- Received April 18, 2021

Revised May 26, 2021

Accepted June 6, 2021

- Corresponding author: Byung Yoon Choi

Department of Otorhinolaryngology-Head and Neck Surgery, Seoul

National University Bundang Hospital, Seoul National University College

of Medicine, 82 Gumi-ro 173beon-gil, Bundang-gu, Seongnam 13620,

Korea

Tel: +82-31-787-7406, Fax: +82-31-787-4057

E-mail: choiby2010@gmail.com

\section{INTRODUCTION}

Malformations of the inner ear account for approximately $20 \%$ of cases of congenital sensorineural hearing loss [1]. In light of the growing body of evidence that cochlear implantation (CI) can be a relatively safe and effective treatment for inner ear malformations, the indications for the use of $\mathrm{CI}$ have expanded, although considerable attention is needed to handle challenging

Copyright ( $) 2021$ by Korean Society of Otorhinolaryngology-Head and Neck Surgery.

This is an open-access article distributed under the terms of the Creative Commons Attribution Non-Commercial License (https://creativecommons.org/licenses/by-nc/4.0)

which permits unrestricted non-commercial use, distribution, and reproduction in any medium, provided the original work is properly cited. 
situations $[2,3]$. Currently, patients with severe inner ear malformations, such as incomplete partition type I (IP-I), cochlear hypoplasia $(\mathrm{CH})$, common cavity, and even cochlear aplasia with a dilated vestibule (CADV), are considered potential candidates for CI [2-4]. However, not all studies fully support this, probably due to the markedly reduced and unpredictable location of the spiral ganglion neurons (SGNs) in cases of severe inner ear anomalies $[5,6]$. Furthermore, the frequent anomalous course of the facial nerve and incapacitating cerebrospinal fluid (CSF) gushers may preclude the electrode array from being positioned correctly [7]. This point aligns with recent clinical studies suggesting that the $\mathrm{CI}$ outcomes for patients with malformed cochleae are heterogeneous, albeit mostly satisfactory, and that this heterogeneity may be largely associated with the status of residual SGNs and the final position of the electrode [8].

In current practice, straight arrays with circumferential electrodes (i.e., full-banded electrodes) are widely used to stimulate residual neural nerve fibers as much as possible in inner ear malformations such as IP-I $[2,3,8]$. The rationale behind using fullbanded electrodes in severely malformed cochleae is that the SGNs are present exclusively at the periphery in severely malformed cochleae without partitions, specifically in the case of the common cavity $[9,10]$. The unpredictable location of the concentration of residual SGNs in such malformations argues against obligatorily attempting to use full-banded electrodes in all cases. Therefore, there is currently no consensus on the ideal electrode for severely malformed cochleae, except in cases of common cavity, CADV, and IP-3, where there is a total deficiency of the modiolus.

Magnetic resonance (MR) imaging could indicate whether the cochlear nerve and modiolus are present; however, it is practically impossible to differentiate total from subtotal modiolus absence in cases of severe malformations. If the nerve and modiolus are shown to be extremely small, but still existent, at least some

\section{H I}

- We present our experience of electrically evoked compound action potential (ECAP) and radiography-based selection of an appropriate electrode for severely malformed cochleae.

- A full-banded straight electrode and slim modiolar electrode were inserted sequentially in a single subject to compare ECAP responses and electrode position.

- Slim modiolar electrodes could be a better fit for cases with a severely deficient but not completely defective modiolus.

- The practice of sticking to full-banded straight electrodes may not always be optimal for cases of incomplete partition type I and cochlear hypoplasia type II.

- An ECAP and radiography-based strategy to identify an appropriate electrode may be useful for severely malformed cochleae, leading to enhanced functional outcomes. proportion of residual SGNs is likely to be present near the modiolar side. In this case, slim modiolar electrodes with the best modiolar proximity would potentially lead to favorable outcomes [11].Theoretically, modiolar-hugging electrodes such as slim modiolar electrodes might be eligible and further outperform the lateral straight electrodes or full-banded electrodes in inner ear malformation cases with a small number of SGNs still present in the modiolus. Given the uncertain distribution of SGNs in IP-I or $\mathrm{CH}$, unlike malformations with a total defect of the modiolus, the question arises of how we can select the ideal electrode type for such cases.

The electrically evoked compound action potential (ECAP) response is largely associated with SGNs [12]. Further, it has been proven that the electrode-modiolus distance is closely correlated with the ECAP thresholds of electrodes, including slim modiolar electrodes [13]. Thus, it is now possible to see immediate feedback of the electrode position with the neural structure. As such, comparing the ECAP thresholds between two electrodes located at both extremes-full-banded straight and slim modiolar electrodes-would help to obtain information on where the majority of SGNs reside in severely malformed cochleae.We hypothesized that, based on the ECAP profile, slim modiolar electrodes with the best modiolar proximity may be a better fit for IP-I or CH if a smaller number of SGNs are present near the modiolus than near the lateral wall. Here, we present our experience of ECAP and radiography-based selection of an appropriate electrode type for three patients with severely malformed cochleae (IP-I, CH, and CADV) for future reference.

\section{MATERIALS AND METHODS}

The Institutional Review Board of the Seoul National University Bundang Hospital (IRB No. IRB-B-2020-211) approved this study, which was conducted in line with the Declaration of Helsinki. We obtained written informed consent from either the parents of children or the participants themselves in this study.

\section{Participants}

We conducted a retrospective review of three patients with severely malformed cochlea who underwent CI between January 2020 and September 2020 at Seoul National University Bundang Hospital. Based on the Sennaroglu classification [14,15], CH type 2 (CH-II, cystic hypoplastic cochlea), IP-I (cystic cochleovestibular malformation), and CADV were included. One patient (CH-II) who previously underwent $\mathrm{CI}$ using the conventional lateral straight electrode (CI422; Cochlear, Australia) at another center, achieved unsatisfactory performance underwent revision surgery.

\section{Electrically evoked compound action potential}

Neural telemetry recordings were performed under sterile con- 
ditions in the operating field. ECAP thresholds were measured in every channel in all subjects using automatic neural response telemetry intraoperatively. To test our hypothesis, we conducted comparative analyses of the ECAP thresholds between the two electrodes were conducted. One surgeon (BYC) conducted all procedures; a straight array with a full-banded electrode (CI24RE [ST]; Cochlear) and slim modiolar electrodes (CI632; Cochlear) were alternately inserted and tested. ECAP thresholds were measured in every channel at a $250 \mathrm{~Hz}$ stimulation rate and 35 sweeps; those that did not elicit any response even at the maximum stimulus were noted as 255 current levels [11]. Between the two different electrodes, the ECAP thresholds in every channel and the number of channels compatible with the eligible response of such subjects were used for comparison.

\section{Post-insertion imaging}

To confirm the exact position of the electrode array, we compared the spiral configuration of the electrode arrays in situ on transorbital X-ray in real-time intraoperatively. In particular, in one patient (case 1, aged 14 years at $\mathrm{CI}$ ), high-resolution temporal bone computed tomography (CT) scans were performed on the day after the surgery using $120 \mathrm{kV}, 64 \times 0.6 \mathrm{~mm}$ collimation, 1 -second rotation, pitch factor of 0.85 , and $205 \mathrm{mAs}$, as per the age of the participants by 256-channel multi-detector CT (SOMATOM Force; Siemens Healthineers, Forchheim, Germany). To view the neural-modiolus interface at every electrode contact, high-resolution temporal bone CT images were reformatted to create the cochlear view following the multiplanar reconstruction algorithm with Ur81 (high-resolution index, resolution index of 19.7line-pair).

\section{Auditory performance}

Speech perception performance was assessed using the categories of auditory performance (CAP) scores preoperatively and postoperatively. CAP is a validated and widely used parameter of auditory receptive ability [16], characterized by a hierarchical scale for children's developing auditory abilities according to eight categories from 0 to 7 . In this study, CAP scores obtained at 3 and 6 months postoperatively were utilized in the outcome anal- ysis, whenever available. In addition, the Infant-Toddler Meaningful Auditory Integration Scale (IT-MAIS) score [17], a structured interview provided by the child's parents, was evaluated preoperatively and postoperatively to assess the child's spontaneous responses to sound in everyday situations. The IT-MAIS score ranged from 0 to 40 . We also obtained pre- and postoperative comparative data of speech perception performance through word and sentence-recognition tasks at $70 \mathrm{~dB}$ SPL in an audioonly condition, particularly in patient 1 (aged 14 years at CI). Receptive and expressive language performance was additionally evaluated in patient 2 (aged 10 months at $\mathrm{CI}$ ) and patient 3 (aged 12 months at CI), as appropriate.

\section{RESULTS}

\section{Demographics}

The clinical characteristics and demographics of all participants are shown in Table 1 . Two of the three patients were male, and their age at $\mathrm{CI}$ ranged from 10 months to 14 years. The audiological results demonstrated that two patients (cases 2 and 3) had no auditory brainstem response and a steady-state auditory response preoperatively, while one patient (case 1) showed bilateral severe-to-profound sensorineural hearing loss without functional residual hearing on preoperative pure-tone audiometry. Two patients (cases 2 and 3) underwent bilateral CI, whereas one patient (case 1 ) received unilateral $\mathrm{CI}$ due to severe cochlear ossification on the contralateral side. In the contralateral ear of the first patient (case 1), significant residual hearing almost reaching 30-40 dB PTA was maintained until the age of 10 and then sudden sensorineural hearing loss developed on that side, leading to both sensorineural hearing loss. Apart from one ear with CADV where the electrode array was inserted via the labyrinthotomy approach, all other electrode arrays were introduced via an extended round window or cochleostomy approach. In this study, all patients experienced a CSF gusher during surgery, except case 3 (left ear, IP-I with normal cochlear nerve). In most cases, a significant decrease or arrest of the CSF gusher was observed during the second electrode insertion. Overall, the CSF

Table 1. Demographic and clinical characteristics of the patients presented herein

\begin{tabular}{|c|c|c|c|c|c|c|c|c|}
\hline Case & Age at $\mathrm{Cl}$ & Sex & $\begin{array}{l}\text { Radiological } \\
\text { demonstration }\end{array}$ & $\mathrm{Cl}$ side & $\begin{array}{l}\text { Electrode insertion } \\
\text { approach }\end{array}$ & $\begin{array}{l}\text { CSF } \\
\text { gusher }\end{array}$ & $\begin{array}{l}\text { Final } \\
\text { electrode }\end{array}$ & $\begin{array}{l}\text { FN stimulation } \\
\text { (switch-on) }\end{array}$ \\
\hline Subject 1 (first Cl) & $11 \mathrm{yr}$ & $M$ & $\mathrm{CH}-\mathrm{Il}$ with $\mathrm{CND}$ & $\mathrm{Lt}$ & Cochleostomy & $(+)$ & $\mathrm{Cl} 422$ & $(-)$ \\
\hline Subject 1 (revision $\mathrm{Cl}^{\text {a)}}$ ) & $14 \mathrm{yr}$ & & $\mathrm{CH}-\mathrm{Il}$ with CND & $\mathrm{Lt}$ & Cochleostomy & $(+)$ & $\mathrm{Cl} 24 \mathrm{RE}(\mathrm{ST})$ & $(-)$ \\
\hline \multirow[t]{2}{*}{ Subject 2} & $10 \mathrm{mo}$ & $\mathrm{F}$ & IP-I with CND & Rt & Cochleostomy & $(+)$ & Cl632 & $(-)$ \\
\hline & & & IP-I with CND & $\mathrm{Lt}$ & Extended RW & $(+)$ & Cl632 & $(-)$ \\
\hline \multirow[t]{2}{*}{ Subject 3} & $12 \mathrm{mo}$ & M & CADV & Rt & Labyrinthotomy & $(+)$ & $\mathrm{Cl} 24 \mathrm{RE}(\mathrm{ST})$ & $(-)$ \\
\hline & & & IP-I with normal CN & $\mathrm{Lt}$ & Extended RW & $(-)$ & Cl632 & $(-)$ \\
\hline
\end{tabular}

$\mathrm{Cl}$, cochlear implant; CSF, cerebrospinal fluid; FN, facial nerve; $\mathrm{CH}$, cochlear hypoplasia; CND, cochlear nerve deficiency; IP-I, incomplete partition type I; CADV, cochlear aplasia with a dilated vestibule; CN, cochlear nerve; Lt, left; Rt, right; RW, round window.

a) Subject 1 underwent a revision $\mathrm{Cl}$ operation 3 years after the first $\mathrm{Cl}$ due to unsatisfactory performance and incomplete insertion. 
leak was well controlled in all cases of CSF gushers. However, otology surgeons should be prepared for CSF gushers during surgery in cases with severely malformed cochleae, considering the increased risk of CSF gushers in the total or partial defect of the modiolus. Additionally, we did not note any difficulty of insertion of each electrode array in any of the patients.

\section{Choice of electrodes and changes in auditory performance}

Case 1 (CH-II with cochlear nerve deficiency) had previously undergone $\mathrm{CI}$ using a conventional lateral straight electrode (CI422) at another center (Fig. 1A). Before revision surgery, ECAP responses were achieved only from four electrodes due to incomplete insertion (Fig. 1A and B), leading to unsatisfactory performance. A revision CI was performed, explanting the CI422 and re-inserting the CI24RE(ST) and CI632 in order one by one. At revision surgery, the CI24RE(ST) array was inserted, and then the ECAP was recorded; next, the array was removed and replaced with the CI632 and the ECAP measurement was repeated with the second electrode array for comparison. Although both electrodes (CI24RE(ST) and CI632) elicited reliable ECAP responses, CI24RE(ST) showed overall lower ECAP thresholds than CI632, except for the distal electrodes (electrodes 1-3) (Fig. 1B). Furthermore, CI632 could not be correctly positioned as would be possible in a normal cochlea, whereas better positioning without kinking and curling of the CI24RE(ST) was observed. Thus, CI24RE(ST) was finally chosen for this case (Fig. 1C), and the completely inserted CI24RE(ST) was shown to outperform the CI422 that had been in situ for 3 years in just 3 months after the revision procedure. Compared to the patient's preoperative status, the sentence perception score was improved by approximately 4 and 22 points at postoperative 3 and 6 months, respectively (Table 2). The patient subjectively felt that the sound quality was better than before revision surgery even at 3 months.

In case 2 (IP-I with cochlear nerve deficiency) (Fig. 2A), CI632 was chosen as it elicited superior ECAP responses when compared with CI24RE(ST), although the responses were mostly unsatisfactory. On post-insertion radiographs, both showed the intended positioning (Fig. 2B and C). Patient 2 showed markedly improved auditory performance postoperatively, considering the accompanying cochlear nerve deficiency. The CAP score improved by 1 (from 0 to 1 ) and 3 (from 0 to 3), and IT-MAIS improved by 18 points (from 6 to 24) and 26 points (from 6 to

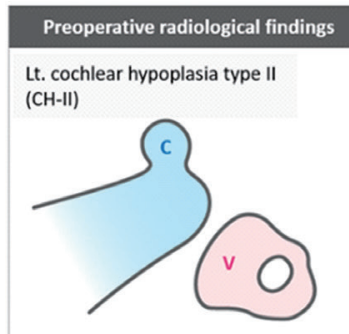

Temporal bone CT: revision case

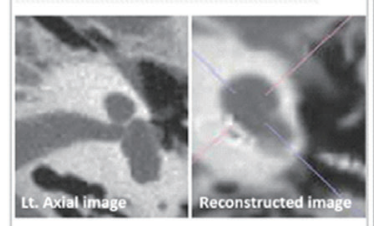

MR imaging: $\mathrm{CN}$ integrity

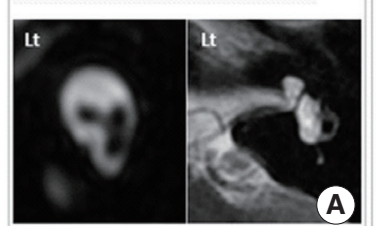

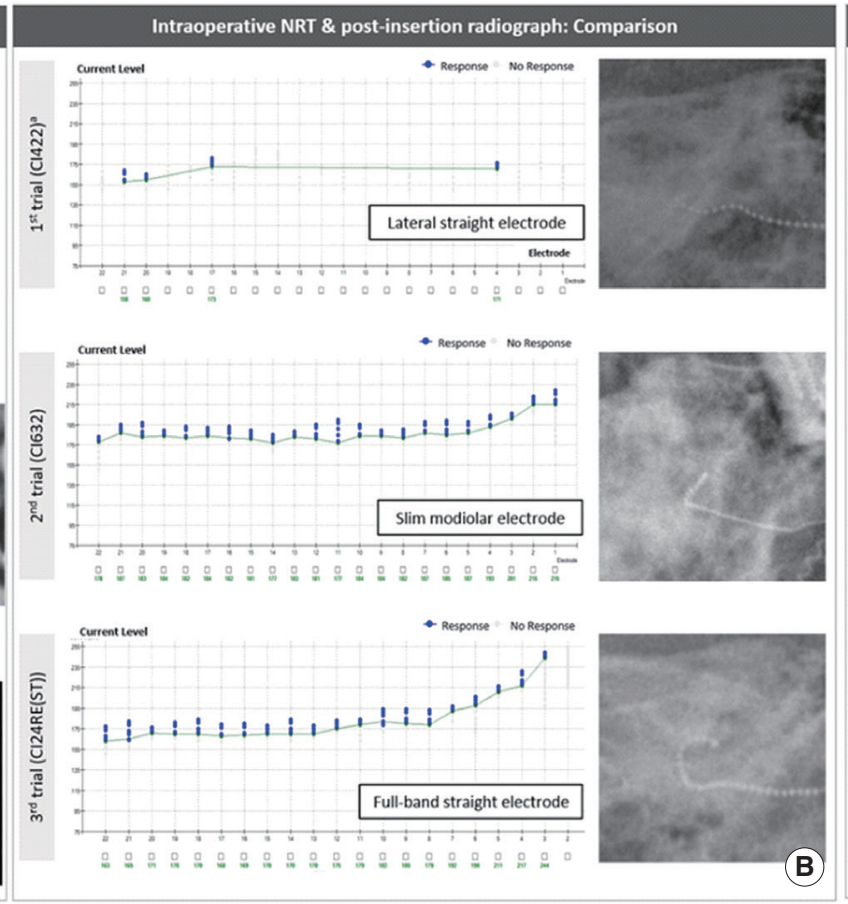

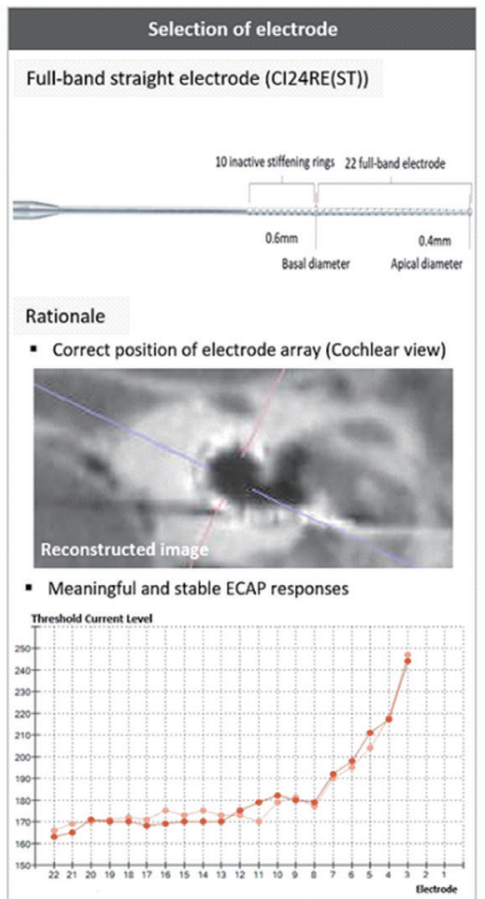

(c)

Fig. 1. Radiological demonstration and electrically evoked compound action potential (ECAP) threshold-based selection of the electrode in the case (case 1) with cochlear hypoplasia type II (CH-II). (A) Schematic illustration showing $\mathrm{CH}-\mathrm{II}$ (C, cochlea; V, vestibule). Temporal bone computed tomography (CT) displays that the cochlea affected by $\mathrm{CH}$-II has smaller dimensions with defective modiolus and interscalar septa, but a normal external outline. On parasagittal oblique magnetic resonance (MR) images, cochlear nerve (CN) deficiency was observed. Based on reformatted high-resolution temporal bone CT images using multiplanar reconstruction algorithm (i.e., cochlear view), the previously implanted conventional lateral straight electrode (Cl422) was incompletely inserted. (B) Initially, ECAP threshold responses were achieved only from four electrodes among the entire Cl422 electrode array that had been incompletely inserted. ECAP thresholds and electrode array position on post-insertion radiographs were evaluated for both Cl24RE(ST) and Cl632. (C) Final selection of the electrode to be inserted for this case. Lt, left; NRT, neural response telemetry. 
32) at postoperative 3 and 6 months, respectively (Table 2).

In the right ear of case 3 (CADV and normal cochleovestibular nerve entering a dilated vestibule) (Fig. 3A), CI632 inserted via a labyrinthotomy approach did not elicit an ECAP response, thereby confirming the incompatibility of the slim modiolar electrode with CADV. In contrast, CI24RE(ST) led to a substantially meaningful ECAP response with correct positioning on post-insertion radiographs after an attempt to reposition the

Table 2. Preoperative and postoperative auditory performance

\begin{tabular}{|c|c|c|c|c|c|c|}
\hline Case & Age at $\mathrm{Cl}$ & Diagnosis & $\mathrm{Cl}$ status & Preoperative & Postoperative $3 \mathrm{mo}$ & Postoperative $6 \mathrm{mo}$ \\
\hline \multirow[t]{4}{*}{ Patient 1} & $14 \mathrm{yr}$ & Lt CH-II with CND & Unilateral $\mathrm{Cl}$ & CAP: 5 & CAP: 5 & CAP: 7 \\
\hline & & & Lt: Cl24RE(ST) & IT-MAIS: 35 & IT-MAIS: 36 & Word (Spondee) scores: 61.11 \\
\hline & & & & Word perception: 35 & Word perception: 35 & $\begin{array}{l}\text { Word (Phonetically Balanced) } \\
\text { scores: } 80\end{array}$ \\
\hline & & & & $\begin{array}{l}\text { Sentence perception: } \\
76.66\end{array}$ & Sentence perception: 80 & $\begin{array}{l}\text { Sentence (Korean central insti- } \\
\text { tute for deafness) scores: } 98\end{array}$ \\
\hline \multirow[t]{4}{*}{ Patient 2} & $10 \mathrm{mo}$ & Rt IP-I with CND & Bilateral $\mathrm{Cl}$ & CAP: 0 & CAP: 1 & CAP: 3 \\
\hline & & Lt IP-I with CND & Rt: Cl632 & IT-MAIS: 6 & IT-MAIS: 24 & IT-MAIS: 32 \\
\hline & & & Lt: Cl632 & Receptive: 2 & Receptive: 7 & Receptive: 11 \\
\hline & & & & Expressive: 4 & Expressive: 10 & Expressive: 10 \\
\hline \multirow[t]{4}{*}{ Patient 3} & $12 \mathrm{mo}$ & Rt CADV & Bilateral $\mathrm{Cl}$ & CAP: 1 & CAP: 3 & NA \\
\hline & & Lt IP-I with normal CN & Rt: Cl24RE(ST) & IT-MAIS: 15 & IT-MAIS: 29 & \\
\hline & & & Lt: Cl632) & Word perception: 5 & Word perception: 24 & \\
\hline & & & & Sentence perception: 13 & Sentence perception: 18 & \\
\hline
\end{tabular}

Cl, cochlear implant; Lt, left; CH-II, cochlear hypoplasia type II; CND, cochlear nerve deficiency; CAP, categories of auditory performance; IT-MAIS, InfantToddler Meaningful Auditory Integration Scale; Rt, right; IP-I, incomplete partition type I; CADV, cochlear aplasia with a dilated vestibule; NA, not available; $\mathrm{CN}$, cochlear nerve.
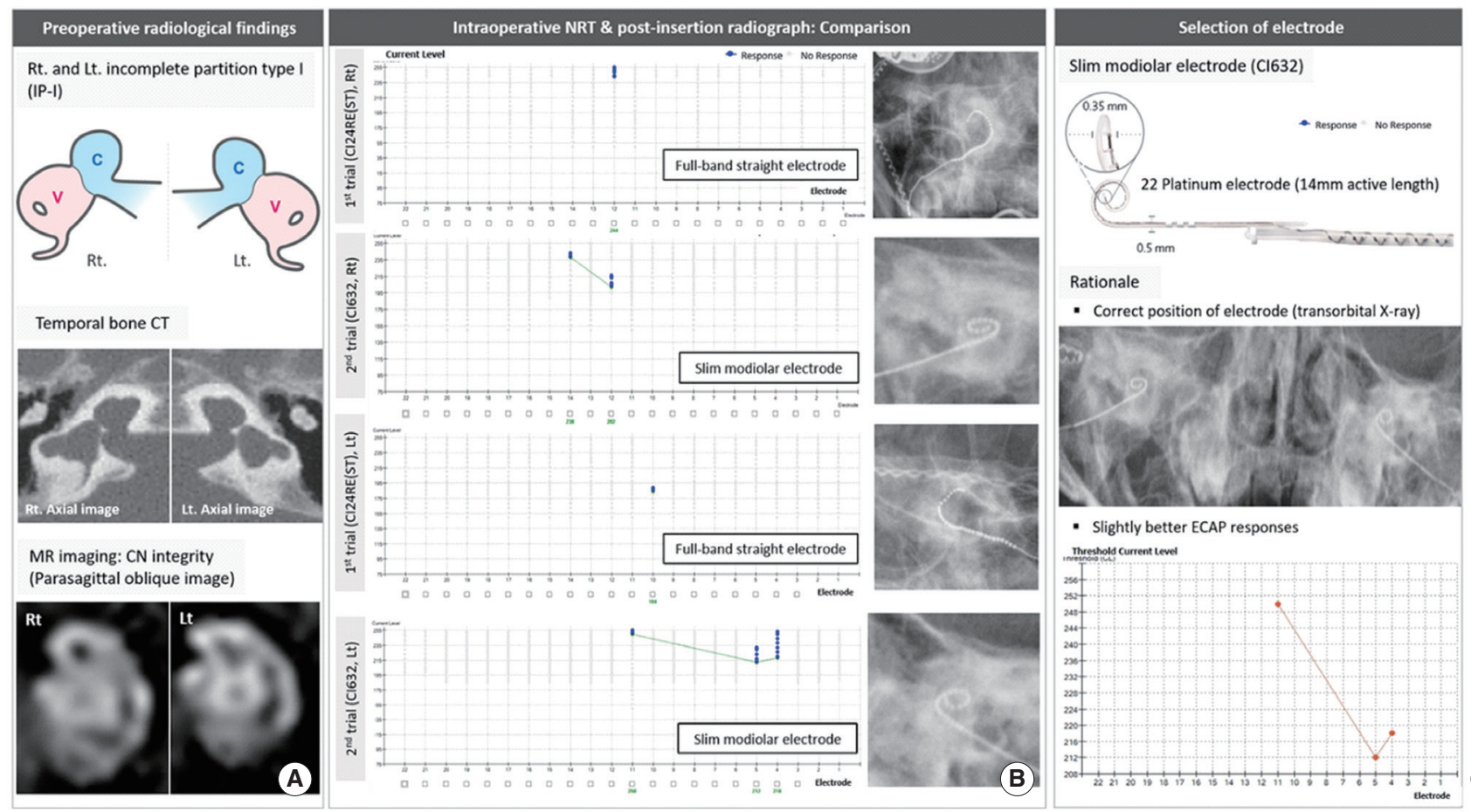

(C)

Fig. 2. Radiological demonstration and electrically evoked compound action potential (ECAP) threshold-based selection of the electrode in the case (case 2) with incomplete partition type I (IP-I). (A) Schematic illustration showing the IP-I anomaly (C, cochlea; V, vestibule). Temporal bone computed tomography (CT) shows a clear differentiation between the cochlea and vestibule, demonstrating the cochlea without a modiolus and interscalar septa, accompanied by a dilated vestibule. On parasagittal oblique magnetic resonance (MR) images, the cochlear nerve $(\mathrm{CN})$ may be deficient on both ears. (B) Sequential trials for selecting ideal electrode: comparison of ECAP thresholds and electrode array position on post-insertion radiographs between the Cl24RE(ST) and Cl632. (C) Final selection of the electrode to be inserted for this case. Rt, right; Lt, left; NRT, neural response telemetry. 

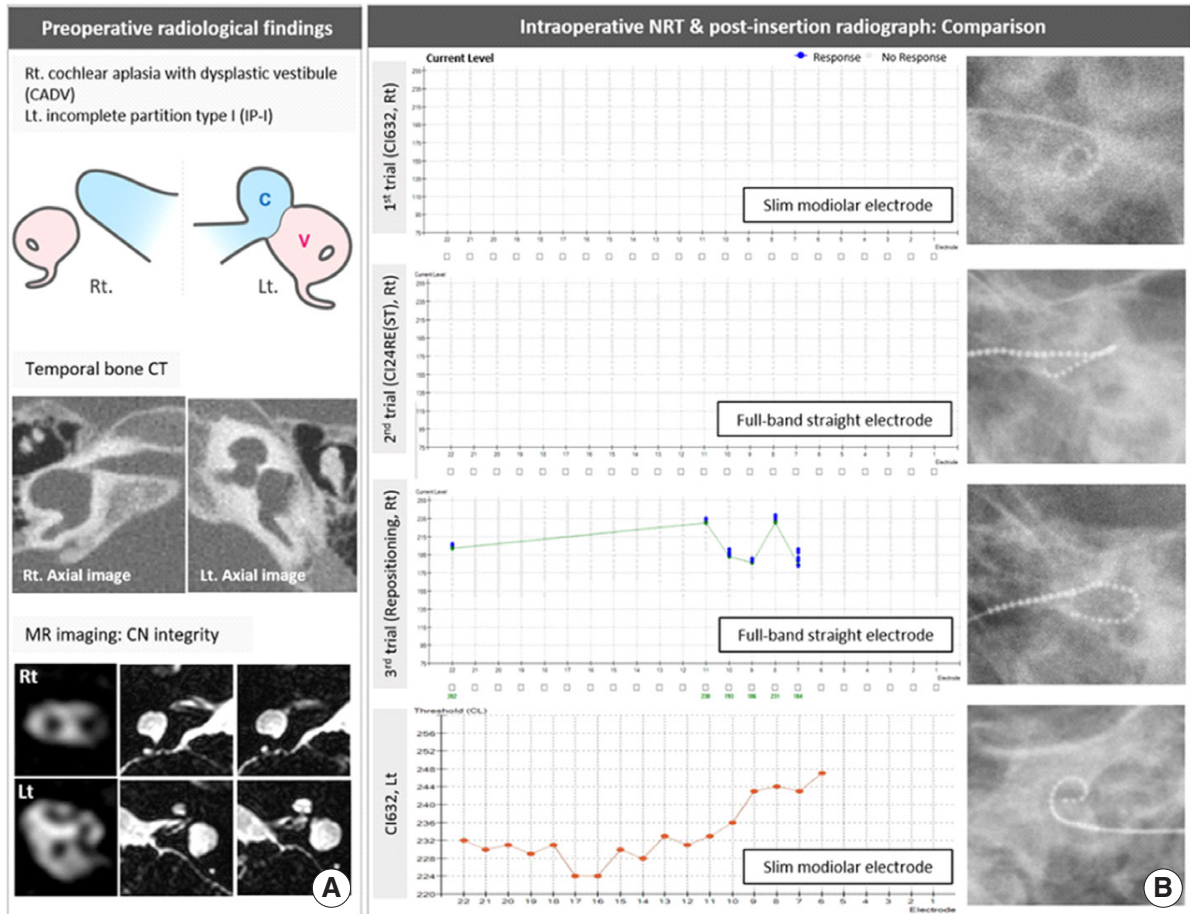

Full-band straight electrode (CI24RE(ST))

\section{Rationale}

- Correct position of electrode array

- Markedly better ECAP responses

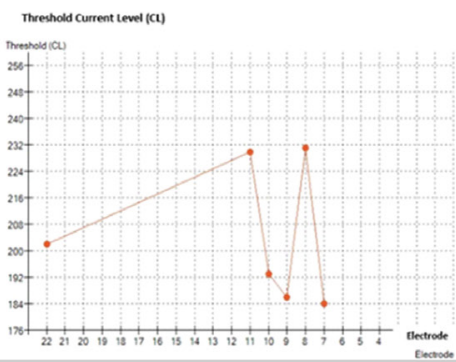

(C)

\section{Selection of electrode (Left ear, IP-I)}

Slim modiolar electrode (CI632)

Rationale

- Correct position of electrode array

- Meaningful and stable ECAP responses

Fig. 3. Radiological demonstration and electrically evoked compound action potential (ECAP) threshold-based selection of the electrode in the case (case 3) with cochlear aplasia with a dilated vestibule (CADV; right [Rt] ear) and incomplete partition type I (IP-I; left [Lt] ear). (A) Schematic illustration showing (CADV, Rt ear) and IP-I (Lt ear) (C indicates cochlea; V indicates vestibule). Temporal bone computed tomography (CT) exhibits a vestibular dilatation (Rt ear) located at the posterolateral part of the fundus, representing CADV. The cochlear nerve (CN) is absent on parasagittal oblique magnetic resonance (MR) images, but notably, the cochleovestibular nerve entering a dilated vestibule was observed on heavily T2-weighted axial images. (B) Sequential trials for selecting the ideal electrode: Comparison of ECAP thresholds and electrode array position on post-insertion radiographs between the Cl24RE(ST) and Cl632. (C, D) Final selection of the electrode to be inserted for this case. NRT, neural response telemetry.
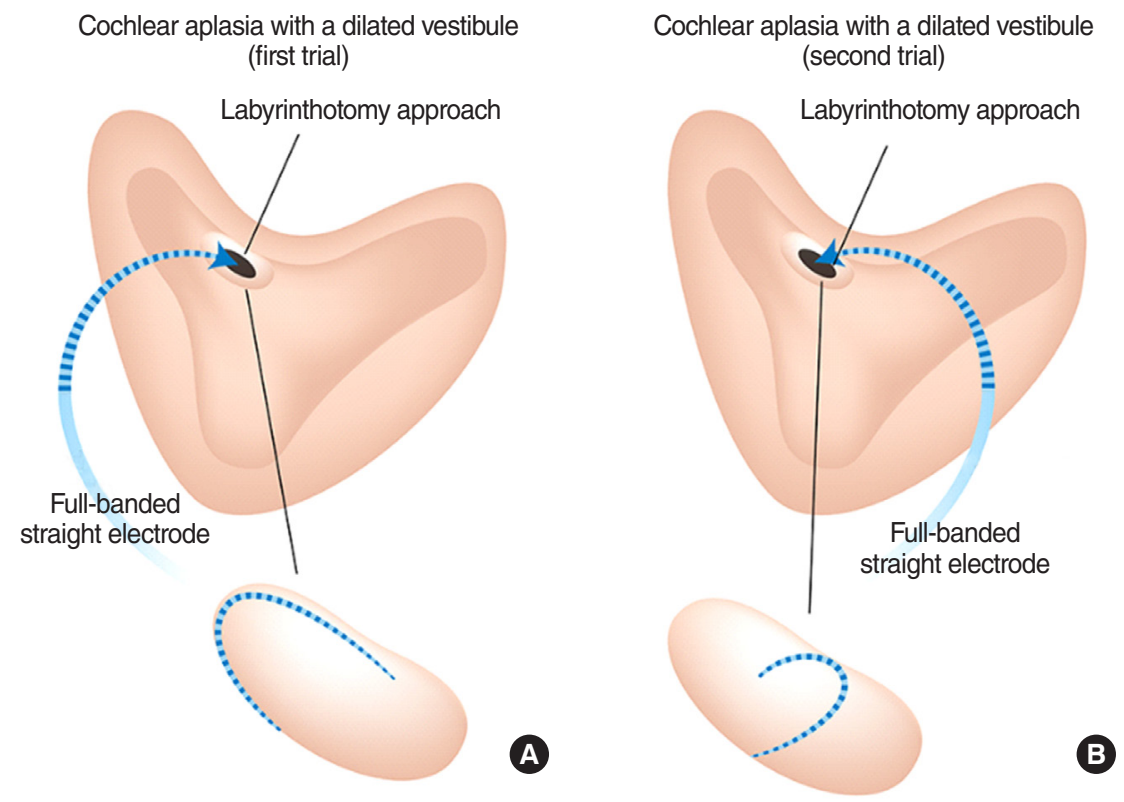

Fig. 4. A schematic diagram of repositioning the full-banded straight electrode array in the cochlear aplasia with a dilated vestibule. (A) Insertion direction parallel to the lateral wall via a labyrinthotomy approach. (B) Insertion direction perpendicular to the lateral wall via a labyrinthotomy approach. 
electrode array (Fig. 3B). As shown in Fig. 4, CI24RE(ST) inserted and positioned parallel to the lateral wall did not elicit the ECAP response, whereas positioning the electrode perpendicular to the lateral wall led to a better ECAP response. Thus, CI24RE(ST) was finally chosen for this right ear with CADV (Fig. 3C). Subsequently, the CI632 electrode was re-inserted into the left ear (IP-I with normal cochlear nerve), leading to significant ECAP responses with correct positioning (Fig. 3B and D). Intriguingly, a CSF gusher was not noted in the left ear. Overall, auditory performance improved considerably from the early postoperative period. The CAP score improved by 2 (from 1 to 3 ) and IT-MAIS improved by 14 points (from 15 to 29 ) at 3 months postoperatively (Table 2).

\section{DISCUSSION}

To the best of our knowledge, we introduced an ECAP-based selection strategy for an appropriate electrode for severely malformed cochleae in this study for the first time. MR imaging could clarify to some extent whether the cochlear nerve and the modiolus are present; however, it is practically impossible to differentiate total from subtotal modiolus absence in such cases. Conversely, the ECAP-based approach was extrapolated to indirectly assess whether SGNs mainly lie in a severely deficient, but not completely defective modiolus, or along the periphery of the cochlear wall in severely malformed cases. As such, a comparative ECAP-based approach measured from the two extreme electrodes inserted sequentially in a single participant would be a feasible way to localize the neural structures in such cases.

In this study, the usefulness of our ECAP-based approach was verified through the most extreme case for which we know the answer. In CADV completely lacking the cochlea and modiolus, the slim modiolar electrode did not elicit any response at all. This strengthens the notion that perimodiolar electrodes, including slim modiolar electrodes, are contraindicated in cases of severe malformations entirely lacking a cochlear aperture and modiolus, such as common cavity and CADV. Unfortunately, it is unclear how positioning the electrode perpendicular to the lateral wall led to a better ECAP response in such a case (see case 3). Nonetheless, our data suggest that neural tissues, including cochlear nerve fibers, are likely to be present-although randomly distributed-along the periphery of the CADV $[9,10,18]$. Efforts may be required to attempt to insert the electrode through a different trajectory, which in turn may elicit favorable language development from CI, even in cases with CADV. Similarly, a previous study, coupled with our functional data, showed that pediatric patients with CADV could also achieve favorable language development from CI using circumferentially stimulating electrodes [4].

The comparative electrophysiological evidence presented herein suggests that modiolar-hugging electrodes could be applicable to severely malformed cochleae if SGNs are presumed to be present, albeit severely defective, near the modiolus. More specifically, in IP-I and CH-II, unlike in CADV, a slim modiolar electrode elicits meaningful ECAP responses, sometimes even better than the full-banded straight electrode in the same patient. Our results suggest that a subset of electrically excitable neural structures is likely to be present around the modiolar region in IP-I and $\mathrm{CH}$-II cases. Importantly, our results imply that the practice of sticking only to the full-banded straight electrode may not always be optimal for such cochlear malformations. Supporting this, previous histopathologic studies have demonstrated that the basal part of the modiolus is present in CH-II [19]. Furthermore, the majority of IP-I specimens demonstrate the presence of a subtotal modiolar defect, but not complete absence [19], demonstrating that these $\mathrm{CI}$ recipients with IP-I or $\mathrm{CH}-\mathrm{II}$ could also benefit from modiolar-hugging electrodes. Indeed, CI632 was finally chosen for IP-I cases (patient 2, both ears; patient 3, left ear) in this study, who showed markedly improved performance at 3 months postoperatively. Contrary to our hypothesis, most cases of IP-I with a deficient modiolus have been implanted with full-banded straight electrodes thus far in the literature $[2,15,20]$. The reason why full-banded straight arrays are preferred over modiolar-hugging electrodes in such cases remains elusive; however, a potential explanation may be the limited number of cases with a curled configuration of the perimodiolar electrode in the cochlea [2]. Overall, slim modiolar electrodes may be more appropriate than full-banded straight electrodes, if a small number of cochlear nerve fibers and SGNs around the rudimentary modiolus are the only electrically excitable structures, even though we lack longer-term speech performance.

As seen in patient 1 and patient 2, testing both types of electrodes (full-banded straight and slim modiolar electrodes) in the same cochlea to compare the ECAP thresholds and radiographybased electrode positions inevitably wastes one electrode. Given the financial implications, the ECAP and radiography-based strategy to identify an appropriate electrode is more beneficial in patients with asymmetric cochlear malformations, such as in case 3. Hence, once used, the slim modiolar electrode can be reserved for the contralateral ear with IP-I and CH-II if it does not work for CADV initially. The rationale behind this is that the slim modiolar electrode may be best for IP-I and CH-II.

It is conceivable that two trials of electrode insertion in the same cochlea might increase the risk of neural tissue damage, including cochlear nerve fibers, adversely affecting the CI outcome. The advantages and disadvantages of multiple ECAP and radiography-based electrode insertions warrant further research. Alternatively, a well-designed promontory test that guides residual SGN preoperatively may serve as a supplement to the ECAP and radiography-based strategy and is expected to help determine the optimal electrode for severely malformed cochleae.

We introduce a novel ECAP-based selection strategy to identify an appropriate electrode for severely malformed cochleae. 
Our results show that the practice of sticking only to the fullbanded straight electrode may not always be optimal for IP-I or $\mathrm{CH}-\mathrm{II}$. Slim modiolar electrodes with the best modiolar proximity could be a better fit for cases with a severely deficient but not completely defective modiolus, eventually leading to significantly enhanced auditory performance.

\section{CONFLICT OF INTEREST}

Byung Yoon Choi is an associate editor of the journal but was not involved in the peer reviewer selection, evaluation, or decision process of this article. No other potential conflicts of interest relevant to this article were reported.

\section{ACKNOWLEDGMENTS}

This research was supported by a grant of the Korea Health Technology R\&D Project through the Korea Health Industry Development Institute (KHIDI), funded by the Ministry of Health \& Welfare, Republic of Korea (grant number: HI20C0285).

This work was supported by grants from Cochlear Korea (062019-298 to BYC) and a research grant through Seoul National University Bundang Hospital (13-2019-002 to BYC).

\section{ORCID}

Sang-Yeon Lee

https://orcid.org/0000-0003-3566-8708

Byung Yoon Choi

https://orcid.org/0000-0001-5125-2118

\section{AUTHOR CONTRIBUTIONS}

Conceptualization: all authors. Data curation: all authors. Formal analysis: SYL. Funding acquisition: BYC. Methodology: BYC. Project administration: SYL.Visualization: SYL.Writing-original draft: SYL.Writing-review \& editing: BYC.

\section{REFERENCES}

1. Jensen J. Malformations of the inner ear in deaf children: a tomographic and clinical study. Acta Radiol Diagn (Stockh). 1968:Suppl 286:3.
2. Suk Y, Lee JH, Lee KS. Surgical outcomes after cochlear implantation in children with incomplete partition type I: comparison with deaf children with a normal inner ear structure. Otol Neurotol. 2015 Jan;36(1):e11-7.

3. Eftekharian A, Eftekharian K, Mokari N, Fazel M. Cochlear implantation in 8 incomplete partition type I. Eur Arch Otorhinolaryngol. 2019 Oct;276(10):2763-8.

4. Jeong SW, Kim LS. Cochlear implantation in children with cochlear aplasia. Acta Otolaryngol. 2012 Sep;132(9):910-5.

5. Schmidt JM. Cochlear neuronal populations in developmental defects of the inner ear: implications for cochlear implantation. Acta Otolaryngol. 1985 Jan-Feb;99(1-2):14-20.

6. Nadol JB Jr. Patterns of neural degeneration in the human cochlea and auditory nerve: implications for cochlear implantation. Otolaryngol Head Neck Surg. 1997 Sep;117(3 Pt 1):220-8.

7. Sennaroglu L, Sarac S, Ergin T. Surgical results of cochlear implantation in malformed cochlea. Otol Neurotol. 2006 Aug;27(5):615-23.

8. Berrettini S, Forli F, De Vito A, Bruschini L, Quaranta N. Cochlear implant in incomplete partition type I. Acta Otorhinolaryngol Ital. 2013 Feb;33(1):56-62.

9. Graham JM, Phelps PD, Michaels L. Congenital malformations of the ear and cochlear implantation in children: review and temporal bone report of common cavity. J Laryngol Otol Suppl. 2000;25: $1-14$.

10. Eisenman DJ, Ashbaugh C, Zwolan TA, Arts HA, Telian SA. Implantation of the malformed cochlea. Otol Neurotol. 2001 Nov;22(6): 834-41.

11. Lee SY, Jung Bae Y, Carandang M, Kim Y, Hee Han J, Huh G, et al. Modiolar proximity of slim modiolar electrodes and cochlear duct length: correlation for potential basis of customized cochlear implantation with perimodiolar electrodes. Ear Hear. 2020 Aug;42(2): 323-33.

12. Briaire JJ, Frijns JH. Unraveling the electrically evoked compound action potential. Hear Res. 2005 Jul;205(1-2):143-56.

13. Lee SY, Han JH, Carandang M, Bae YJ, Choi BY. Simpler and effective radiological evaluations for modiolar proximity of a slim modiolar cochlear implant electrode. Sci Rep. 2020 Oct;10(1):17714.

14. Sennaroglu L, Saatci I. A new classification for cochleovestibular malformations. Laryngoscope. 2002 Dec;112(12):2230-41.

15. Sennaroglu L, Bajin MD. Classification and current management of inner ear malformations. Balkan Med J. 2017 Sep;34(5):397-411.

16. Archbold S, Lutman ME, Marshall DH. Categories of auditory performance. Ann Otol Rhinol Laryngol Suppl. 1995 Sep;166:312-4.

17. Yoon MS. Validity and reliability of the IT-MAIS Korean version in children with normal hearing. Commun Sci Disord. 2011;16(4):494502.

18. Dhanasingh A, Jolly C. An overview of cochlear implant electrode array designs. Hear Res. 2017 Dec;356:93-103.

19. Sennaroglu L. Histopathology of inner ear malformations: do we have enough evidence to explain pathophysiology? Cochlear Implants Int. 2016;17(1):3-20.

20. Kim SY, Kim MB, Chung WH, Cho YS, Hong SH, Moon IJ. Evaluating reasons for revision surgery and device failure rates in patients who underwent cochlear implantation surgery. JAMA Otolaryngol Head Neck Surg. 2020 May;146(5):414-20. 